\title{
Food Safety Training: A Study of Food Handlers Working in Hotels in the North of Jordan
}

\author{
Ma'moun A. Habiballah, PhD. \\ Firas J. Al-Shakhsheer, PhD. \\ Samer M. Al-Sabi, PhD. \\ Mousa A. Masadeh, PhD.
}

Al-Hussein Bin Talal University, Ma'an, Jordan

Doi:10.19044/esj.2018.v14n26p127 URL:http://dx.doi.org/10.19044/esj.2018.v14n26p127

\begin{abstract}
In spite of decenniums of advancement, a numerous foodborne outbreaks continue to occur each year; these were mostly happening due to malpractices of employees working in foodservices. This study aims to investigate the role of food safety training in improving food handlers' practices through the application of Knowledge, Attitude and Practices (KAP) model. Primary data were collected using a self-completed questionnaires that were collected from 100 Food and Beverage (F\&B) employees working in hotels at the north of Jordan. Findings confirmed the role of food safety training in increasing F\&B employees' knowledge which positively correlates with their food handling practices. Furthermore, this study explored work environment elements affecting the transformation of learned knowledge into proper food handling practices; these include management motivation, availability of necessary resources and facilities and reinforcement programs. The study contributed to knowledge by employing the aforementioned variables to produce an adapted version of the KAP model.
\end{abstract}

Keywords: Training, Knowledge, Food handling, Practices.

\section{Introduction:}

Training is a useful tool that enables hospitality organizations to manage many of the challenges (Tracey and Cardenas 1996). As argued by Wilson et al. (1998), training helps to attaining economic achievements, increasing margins of profits, improving skills, reducing levels of turnover and to cope with changeful environment. Hospitality as a field of science is a multidisciplinary one which is established on different hard and dynamic disciplines including mathematics, engineering, chemistry, biology and microbiology (Jones, 2004). Among various hospitality industry operations, 
food services are the manufacturing ones that contain complex processes of a technological and operational nature (Jones \& Lockwood, 2002). Besides complexity of work, food handlers are exposed to many hazards at workplace (such as high temperatures, injuries \& slippery floors), these risks can be managed and reduced by proper supervision and training (Hendricks \& Layne, 1999). The training role in improving work safety was evidenced in a study by Sinclair et al. (2003) which confirmed that safety training can enhance food service employees' knowledge of work safety and decrease their injury rate; study's findings suggested that training is more significant than employees' experience to enhance work safety at foodservice organizations. Furthermore, it is affirmed that one of the major reasons of foodborne outbreaks is the lack of training and education of food handlers (Motarjemi \& Käferstein, 1999; Engel., 2001). The shortage in food handlers' education and training in foodservices contains many risks that are severe to the public health (Seaman \& Eves, 2006). Training of food hygiene plays an essential role in controlling the due diligence of food industry workers (Engel, 1998); also, it demonstrates this due diligence to customers and even to authorities (Engel, 2001).

For the dangerous and complex operations of food handling in the hospitality industry, training of food hygiene is considered as fundamental stepping stone to achieve food safety (Engel, 2001). Food workers' training considered to be the strategy that can upgrade levels of food safety in the long run throughout the food industry (Griffith, 2002). Hence, it becomes a necessity to provide food handlers with at least the basic knowledge of proper food hygiene methods (Guthrie, 1988). In other words, food service employees should be trained to have the knowledge needed in undertaking their tasks and to attain skills improving their hygiene practices of food handling (MacAuslan, 2003); these include teamwork skills, proper food handling practices reducing food hazards, ability to control food crosscontamination and personal hygiene skills (Johns, 1995). Moreover, as MacAuslan (2003) argued, the provision of comprehensive food hygiene training helps food service organizations to gain many benefits: improving of work conditions, achieving better reputation and sales, enhancing customers and employees satisfaction, building good relationships with health and environmental authorities and complying with legal requisites. On the other hand, the lack of food handlers' training may leads to problems for food service establishments including the decrease of employees satisfaction which cause higher turnover, costs of legal penalties, reducing operations' efficiency and effectiveness and the rise of foodborne diseases (Knowles, 2002 \& MacAuslan, 2003).

Some researchers including Arif and Hassoun (1969), Abu Al Saud (1983), Khan et al. (1987), Wakid (2006), Abu-Madi et al. (2008), Simsek et al. (2009) and Imam et al. (2016) have reported many cases of foodborne 
infection among food handlers' in the Middle East; similar cases were also reported in Jordan by Al-Lahham et al. (1990), Khuri-Bulos et al. (1994) and Abdel-Dayem et al. (2014) who suggested food safety training as a method for improving consumers and food handlers practices and increasing protection against foodborne infections in Jordan. Thus, this research sheds more light on the role of food safety training in improving food handlers' practices working on Jordanian hotels. Hotels are major components of the tourism industry. According to Aziri and Nedelea (2013), tourism is the key mean of developing economies in most countries. Jordan has a promising tourism industry; in 2015 it contributed by $11.8 \%$ to the GDP (Jordan Investment Commission (JIC), 2015). It is an important economic sector which needs always more research improving its product; this ultimately enhances the competitiveness of Jordan as a world tourism destination.

\section{Background}

Training is a "planned process to modify attitude or skill behaviour through learning experience to achieve effective performance in an activity or range of activities" (Manpower Services Commission (MSC), 1981, p. 62). Accordingly, training is a process that aims to amend individuals' behaviour to improve the effectiveness of their performance. As observed by Smith (1994), employees who are not aware of reasons and rationale behind doing a certain behaviour, tend to neglect that behaviour or to act improperly; such awareness problem in food handling operations can be overcame by offering an appropriate training to various levels of food handlers. Furthermore, learning of proper food handling enables food handlers to abandon some malpractices adopted traditionally by the effects of culture (Foster 1983). It is believed that ongoing training of food safety plays a significant role in enhancing good food handling practices (Walter et al., 1997). It is generally recognized that higher level of food safety training, leads to better commitment of food safety (Worsfold \& Griffith 2003).

Training tends to change attitudes and practices depending on the boost of knowledge (Ehiri et al., 1997). Most food safety trainings adopt the model of Knowledge Attitudes and Practices (KAP), assuming that the providing of knowledge leads to a change in food handlers' attitudes which consequently will result in a change of their practices (Rennie, 1995). However, the food safety training efficiency in improving food handlers' practices is still debatable as shown in many studies (Mortlock et al., 1999). Many scholars (cf. Tones \& Tilford, 1994 \& Howes et al., 1996) argued that the KAP model application in food handlers training may increases their food safety knowledge, but this knowledge increase alone is not necessarily enough to change their practices; such argument was empirically confirmed by the study of Howes et al., (1996), that was carried out on 69 food handling employees 
working in three universities at Ontario-Canada, which found that food safety training was able to provide food handlers with knowledge about proper food handing practices but without achieving a significant improvement in their practices afterwards Powell et al. (1997) investigated 50 individuals employed for 12 food establishments in the UK where no significant differences were found in knowledge about hygiene between trained workers and non-trained ones; researchers also did not find a relationship between the level of food handlers' knowledge and the standards of hygiene for the studied premises. Similar findings were attained by Walker et al. (2003) who examined 444 food handlers working in 104 food premises; these food handlers were found to have a poor hygiene knowledge although most of them $(57 \%)$ had a formal training in food safety. In a more recent study undertaken by Hertzman and Barrash (2007) on caterers in Las Vegas-USA, a malpractices were observed from study's subjects even though some of them were knowledgeable abut food safety; these malpractices were ascribed to work pressure, shortening in management supervision and the lack of time. This explanation consists with the findings of other scholars (Clayton et al., 2002 \& Green \& Selman, 2005).

A different group of scholars expounded the influence of food safety training in enhancing food safety. As argued by Smith (1994), food hygiene training improves standards of personal hygiene of food handlers and the public. In a comparison of training, knowledge and practices of 75 food handlers working in foodservice premises, Tebbutt (1992) explored that measures of food hygiene practices (e.g. personal hygiene \& contamination risk) were improved significantly in premises adopting training programmes for their staff, especially those who were handling high-risk foods. Thereafter, a survey by Essex Chief Environmental Health Officers' Food Group (ECEHOFG) was undertaken on 156 food establishments to assess the effectiveness of food handlers' training had confirmed the role of staff training in improving the hygienic food handling (Kitcher, 1994). In another study by Kirby and Gardiner (1997) which was carried out on 20 food establishments in the UK, it was noted that food workers who had formal hygiene training demonstrated more hygienic practices than their peers who had none; they also concluded that for food premises to achieve high food hygiene standards, they should adopt food hygiene training as a normal procedures. The previous conclusion was confirmed by Angelillo et al. (2000) who found that most of food handlers with positive attitudes toward the control and prevention of foodborne diseases were those involved in a continuing educational course. Sagoo et al. (2003) carried out a study of 1,502 food retailing and catering premises in the UK within which, deficiencies in cleaning measures and practices were identified in food establishments that do not apply a food hygiene training; the researchers underscored the necessity of training for both 
food handlers and their managers to achieve high levels of cleaning practices and measures.

A further group of authors believe that training of food safety alone is not sufficient to create the required change in food handlers' behaviour into achieving high standards of food safety. It is believed that to achieve a better change in a food handling behaviour, the "settings approach to health promotion" must be adopted (Rennie, 1995, p.79); this means, that a supporting environment must be applied including incentives and frequent reinforcement of new hygiene practices and by providing the necessary facilitation of these practices (Rennie 1994:1995). Nevertheless, empirical evidences found that food businesses in most cases do not plan or/and implement any refresher training (Mortlock et al., 2000 \& Worsfold, 2005); this increases the opportunity of skills decay risk, limiting employees' ability to implement learned skills (Worsfold, 2005). According to Tones and Tilford (1994), knowledge needs to be accompanied with other mechanisms to create positive attitudes and to motivate actions; this was supported by Ray et al. (1997) and Clayton et al. (2002) who demonstrated that training of food hygiene alone is not sufficient to change food handlers' behaviour, they identified other elements to create the required change in food handlers' practices including supportive management culture, availability of resources, feedback of food safety performance and goal setting; of these elements, the feedback on food safety performance helps food handlers to determine aspects of their behaviour that need to be modified (Ray et al., 1997). Moreover, approaches which consider social and environmental effects on food safety need to be adopted instead of relying on training alone as an isolated method to enhance food safety (Ehiri et al., 1997). Hence, the use of food hygiene training in controlling food safety must be adopted as a part within an overall strategy.

In spite the importance of training in enhancing food safety levels, improper training threats food safety more than no training; this is because it provides trainees with misleading and/or incorrect information. Also, it may give food handlers a mistaken sense of security, making them ignore some hazards, supposing that they are educated enough to identify critical hazards (Ackerly, 1989). To guarantee the effectiveness of training on enhancing food safety, certain aspects must be managed successfully in the training experience.

First, the trainers' possession of certain skills that enhance their effectiveness including their ability to grasp materials of learning, the ability to assess and understand trainees' needs, useful communication skills and the capability to build relationship with the trainees (Engel, 2001). Second, the commitment of food service managers to the training on food safety which improves its effectiveness (MacAuslan, 2003); this commitment can be 
increased through a wide promotion of the training benefits to food service managers; such promotion must clarify how food hygiene training helps these mangers to minimising food risks inhered in their businesses (Mortlock et al., 2000). Third, the elements beyond training context that facilitate the transformation of knowledge into practices; examples include workplace characteristics and the relevance and usefulness of training design to trainees' characteristics and to their job (Baldwin \& Ford, 1988). It was argued by Worsfold et al. (2004), that the achievement of hygiene performance is difficult in an environment that lacks facilities necessary to ensure high levels of hygiene. Fourth, the cultural and social aspects of work environment. In a study by Tracey et al. (1995) of 505 US supermarkets managers, a direct relationship was found between trainees' post-training behaviour and the culture of continuous-learning and the supportive climate at their workplace; researchers also demonstrated a positive link between the supportive work environment and the motivation toward subsequent training success. Other work environmental variables that may affect the transmission of trainees' learned skills to their jobs include supervisor authorisation, supervisor and peer support and the availability of aids or constraints at workplace (Seyler et al., 1998)

A further matter which may impact food safety training's quality is the style of training (informal versus formal). In general, Small-to-Medium size Enterprises (SMEs) have a tendency to implement informal (on-the-job) training; this is because of the lack of necessary time to send their employees into a formal training courses (off-the-job training) (Hendry et al., 1992). Such tendency of small food businesses to apply on-the-job training was also confirmed by Pratten and Curtis (2002) and Worsfold (2005). Nevertheless, it is believed that formal training provides greater quality and more consistent than the informal training which is accomplished by managers and/or peers (Manning, 1994). However, despite the differences amongst formal training and the informal one, it is suggested that firms must adopt training programmes enhancing both types of training (Griffith, 2002).

The previous discussion demonstrates that food safety training's influence on food handlers' practices remains a questionable-researched issue; up to date, scholars within this field have proffered results, conclusions and variables concerning training' ability in improving food handler's practices. Scholars who discussed and/or studied training impact on food handlers' behaviour can be clustered into three major groups; the first group (Tebbutt, 1992; Smith, 1994; Kitcher, 1994; Kirby \& Gardiner, 1997; Angelillo et al., 2000 \& Sagoo et al., 2003) consider food safety training as an effective tool to improve food handlers' hygienic behaviour; the second group (Rennie, 1994; Tones \& Tilford, 1994; Luby et al., 1993; Howes et al., 1996; Powell et al., 1997; Walker et al., 2003 \& Hertzman \& Barrash, 2007) argues that in 
spite of food safety training's ability in increasing the knowledge of food handlers, it cannot produce a hygienic performance; finally, the third group (Ray et al., 1997; Ehiri et al., 1997; \& Clayton et al., 2002) believes that food handlers' practices cannot be changed by training alone and they suggest that it must be accompanied by a supportive environment.

Building on previous literature, the relationship between food handlers' practices and food safety training, in a different context (Jordan \& Arabic world) is investigated here. In general, the food safety discipline in Jordan suffers from the paucity of research. Most of the studies investigating food safety topics in Jordan were microbiological ones reporting outbreaks of foodborne diseases (cf. Al-lahham et al., 1990; Khuri-Bulos et al., 1994 \& Abdel-Dayem et al., 2014). However, few researches were found addressing practices of food handlers in Jordan; examples include FAO/WHO (2005), Osaili et al. (2011), Sharif et al. (2013), Osaili et al. (2013) and Habiballah et al. (2017). In spite that all of these studies had covered the association between knowledge of food handlers and their practices, only three of them (Sharif et al., 2013; Osaili et al., 2013 \& Habiballah et al., 2017) were carried out on professional food handlers. Osaili et al. (2013) were focusing only on food handlers' knowledge and factors affecting it; while Sharif et al. (2013) covered the KAP model's constructs. Nevertheless, the current study developed an amended version of the KAP model, within which some intermediating factors were added; these were examined to measure their influence on food safety training effectiveness; also, this study took into consideration the differences between formal versus informal training in affecting food handlers' practices.

\section{Model testing}

A modified KAP model was adopted in this study as a conceptual model. In the light of previous literature review, new variables were merged to the KAP model; these include reinforcement programs, availability of needed resources and facilities and management motivation. The constructs of the current study's model were interrelated by five hypotheses:

Hypothesis 1:

$\mathrm{H}_{0}$ : there is no significant difference in food safety knowledge amongst food handlers who had different levels of food safety training.

$\mathrm{H}_{1}$ : there is a significant difference in food safety knowledge amongst food handlers who had different levels of food safety training.

Hypothesis 2:

$\mathrm{H}_{0}$ : there is no significant relationship between food handlers' food safety knowledge and their food handling practices.

$\mathrm{H}_{1}$ : there is a significant relationship between food handlers' food safety knowledge and their food handling practices. 
Hypothesis 3:

$\mathrm{H}_{0}$ : the motivation of management to implement learned skills of proper food handling has no intervening effect on the relationship between food handlers' practices and the level of their food safety knowledge.

$\mathrm{H}_{1}$ : the motivation of management to implement learned skills of proper food handling has an intervening effect on the relationship between food handlers' practices and the level of their food safety knowledge.

Hypothesis 4:

$\mathrm{H}_{0}$ : programmes of reinforcement for learned skills of proper food handling have no intervening impact on the relationship between food handlers' practices and the level of their food safety knowledge.

$\mathrm{H}_{1}$ : programmes of reinforcement for learned skills of proper food handling have an intervening impact on the relationship between food handlers' practices and the level of their food safety knowledge.

Hypothesis 5:

$\mathrm{H}_{0}$ : the availability of needed resources and facilities to implement learned skills has no intervening impact on the relationship between food handlers' practices and the level of their food safety knowledge.

$\mathrm{H}_{1}$ : the availability of needed resources and facilities to implement learned skills has an intervening impact on the relationship between food handlers' practices and the level of their food safety knowledge.

\section{Methodology}

A designated self-completed closed questionnaire, constituting four major sections, was employed as a data collection tool. The first section measures the level of participant's food safety knowledge; it is using a five multiple-choice questions addressing various aspects of food safety including expected hazards of food (question $1 \& 3$ ), preservation of food (question $2 \&$ 5) and controlling of food hazard (question 4); responses to these questions were manipulated into a single indicator (average) of food safety knowledge. Section two measures F\&B employees' food handling practices using six items of Likert-scale from 1 to 5 (where $1=$ Never; $2=$ Occasionally; $3=$ Sometimes; $4=$ Frequently \& $5=$ Whenever it is necessary); items of the second section were built based on proper food handling practices defined by many scholars (cf. Bryan, 1988; Altekruse et al., 1999; Parish, 1998; Vought \& Tatini, 1998 \& Shapiro et al., 1999). Section three was designed to identify if participants had any food safety training (formal or informal); such identification was accomplished using a multiple-choice question. Furthermore, the third section aimed to assess work-place elements (management motivation, reinforcement programmes $\&$ the availability of resources) that may have an impact on the transmission of food safety knowledge into proper food handling practices; to do so, three close-ended questions were employed to measure these elements. 
The fourth section of the questionnaire identified respondents' demographics including their age group, gender and level of education.

Following a pilot test of 20 food handlers from different hotels in the northern district of Jordan (including three cities: Irbid, Ajlun \& Jarash); fortunately, there was not any amendment to be made for any of the questionnaire's items. Afterward, 110 questionnaires were completed by food handlers working in 23 hotels at the north of Jordan; these were accessed conveniently by the researchers using a delivery and collection technique of distribution. Ten of the collected questionnaires were found unusable for data analysis due to their improper completion; accordingly, they were excluded from data analysis. Questionnaires' reliability was assessed using Cronbach alpha analysis of internal consistency which was good with a Cronbach alpha> 0.7 (cf. Cavana et al. 2000). Using descriptive statistics, the demographic characteristics of study's sample were collected; then, parametric analyses were carried out to test study's hypotheses, including partial correlation and one-way ANOVA.

\section{Results \\ Sample profile}

The majority of the $100 \mathrm{~F} \& \mathrm{~B}$ employee participated in this research were males $(85 \%)$; more than half of the respondents were Jordanians $(88 \%)$. Also, the participants were mostly young; 91\% aged less than 35 years, implying the tendency for Jordanian hotels to employ younger workers in operational jobs. Surprisingly, only $28 \%$ of the participants have a degree. Unfortunately, there have not been any published statistics found about the characteristics of hotels F\&B employees in the north of Jordan. Nevertheless, published statistics of the JMTOA (2017) reveal that the majority (91.5\%) of employees within Jordanian hotels are males and $89.3 \%$ of them are Jordanians; this confirms the representativeness of the current study's sample.

\section{Hypotheses examination}

The current study treats training of food safety as an independent variable that is expected to influence F\&B employees' food handling practices. To explore the aforementioned influence this research model was constructed using the KAP model variables including Knowledge "K" (knowledge of food safety) and Practice "P" (food handlers' practices). Further variables were appended to the research model: programmes of reinforcement for learned skills, management motivation to implement learned skills and availability of necessary resources and facilities to implement learned skills. According to the literature review, these variables were expected to intervene the relationship between food handlers' food safety knowledge and their food handling practices. In other words, the added constructs were expected to 
impact the transformation of learned skills and knowledge of food safety into a good food handling practices.

Five hypotheses were developed to examine the model. The first hypothesis explores the impact of food safety training of $\mathrm{F} \& \mathrm{~B}$ employees on their knowledge of food safety whilst the other four hypotheses were developed to test the relationship connecting F\&B employees' knowledge of food safety and their food handling practices, and to evaluate the intermediating influence of the other variables (management motivation, availability of resources \& facilities \& reinforcement programmes) on this relationship.

To explore the variance in food safety knowledge among F\&B employees who were grouped according to their different levels of food safety training, a one-way ANOVA was employed. As shown in table (1) below, a statistically significant difference was found in F\&B employees' scores of food safety knowledge across all levels (groups) of food safety training where $\mathrm{F}$ (2, $97)=31.90, p \leq 0.001$, confirming the alternative part of hypothesis 1 and rejecting the null one. Furthermore, Table (1) shows the Eta squared value $\left(\eta^{2}\right)$ that indicates the effect size of the difference in training levels on F\&B employees' food safety knowledge; in this study the size of effect was very large (39.6\%) according to the guidelines of Cohen (1988).

Table 1: One-way ANOVA analysis for variance of food safety knowledge over different food safety training levels

\begin{tabular}{|l|l|l|l|l|l|l|}
\hline Group & Mean & SD & F & Df & Sig. & $\eta^{2}$ \\
\hline Formal food safety training & 4.00 & 0.7 & \multirow{2}{*}{31.90} & 2 & 0.000 & 0.396 \\
\cline { 1 - 3 } Informal food safety training & 2.39 & 0.57 & & & & \\
\cline { 1 - 3 } No training & 1.54 & 0.92 & & & & \\
\hline
\end{tabular}

The second section of the study's model cements the relationship between F\&B employees' food safety knowledge and their food handling practices and depicts the role of the reinforcement programmes, management motivation and availability of resources and facilities constructs in intervening the previously mentioned relationship. To explore such relationship and the intervening effect of other model's constructs, hypotheses 2, 3, 4 and 5 were developed; these were examined using a partial correlation analysis. This analysis' results are shown in Table 2: 
Table 2: Partial correlation of the relationship between F\&B employees' food safety knowledge and their food handling practices

\begin{tabular}{|l|l|l|}
\hline Intervening variable & Correlated variable & $\begin{array}{l}\text { Food } \\
\text { handling } \\
\text { practices }\end{array}$ \\
\hline Before controlling management motivation & Food safety knowledge & $0.582^{* * *}$ \\
\hline After controlling management motivation & Food safety knowledge & $0.472 * * *$ \\
\hline $\begin{array}{l}\text { Before controlling availability of facilities and } \\
\text { resources }\end{array}$ & Food safety knowledge & $0.582 * * *$ \\
\hline $\begin{array}{l}\text { After controlling availability of facilities and } \\
\text { resources }\end{array}$ & Food safety knowledge & $0.494 * * *$ \\
\hline Before controlling reinforcement programs programs & Food safety knowledge & $0.582 * * *$ \\
\hline After controlling reinforcement & Food safety knowledge & $0.484 * * *$ \\
\hline
\end{tabular}

As presented in table 2, there is a significant moderate correlation ( $\mathrm{r}$ $=0.582, \mathrm{n}=100, \mathrm{p}<0.001$ ) between $\mathrm{F} \& \mathrm{~B}$ employees' food safety knowledge and their food handling practices; accordingly, the null hypothesis 2 was rejected and the alternative one was accepted. The table above also displays the results of examination for hypotheses 3,4 and 5. A medium positive partial correlation is found between the level of food safety knowledge for F\&B employees, controlling for the motivation of management to apply trained skills in workplace $(\mathrm{r}=0.472, \mathrm{n}=100, \mathrm{p}<0.001)$ and their food handling practices. Another medium positive partial correlation is found between levels of food safety knowledge for $\mathrm{F} \& \mathrm{~B}$ employees, controlling the programmes of reinforcement for trained skills $(r=0.484, n=100, p<0.001)$ and their food handling practices. Finally, a medium positive partial correlation is found between levels of food safety knowledge of F\&B employees, controlling for the availableness of required facilities and resources to apply trained skills ( $\mathrm{r}$ $=0.494, \mathrm{n}=100, \mathrm{p}<0.001)$ and their food handling practices. An inspection of the zero order correlation ( $\mathrm{r}=0.582)$ indicates that controlling for the three aforementioned intervening variables has a strong influence on the strength of correlation between food safety knowledge and food handling practices. In other words, these results reveal that the intermediating variables examined in this study influence the transmission of trained food safety skills into a good food handling practices at the workplace. Consequently, the alternative hypotheses 3,4 and 5 were accepted.

\section{Discussion}

Training increases food handlers' awareness of the workplace practices and motivates them to follow proper food handling practices (Smith 1994). Nevertheless, the food safety training role in improving practices of food handlers remains a questionable matter. Some studies (e.g., Howes et al., 1996; Powell et al., 1997; Walker et al., 2003; Hertzman \& Barrash, 2007) 
concluded that food safety training provision does not improve food handlers' practices, while others (Tebbutt, 1992; Kitcher, 1994; Kirby \& Gardiner, 1997; Angellilo et al., 2000; Sagoo et al., 2003) confirmed the food safety training usefulness in enhancing proper food handling practices. The current study contributes to the debate about the role of food safety training in improving food handlers' practices by investigating that role empirically on F\&B employees working in the Jordanian hotel industry; this investigation went through three phases of analysis:

\section{Phase 1: Analysis for the influence of food safety training on food handlers' food safety knowledge}

In general, it is expected that training can change the behaviour of individuals by increasing their knowledge (Ehiri et al., 1997). Thence, during the first phase of this research the influence of food safety training on F\&B employees' knowledge of food safety was examined. Furthermore, this influence was evaluated over groups of employees according to the type of training they had (informal vs. formal training) using the one-way ANOVA analysis; a significant strong effect of food safety training was apparent on food safety knowledge of F\&B employees. Furthermore, employees who went through a formal food safety training achieved a significantly higher levels of food safety knowledge scores $(M=4.00 ; S D=0.7)$ than those who went through an informal training $(M=2.39 ; S D=0.57)$. These findings consist with previous studies (cf. Naing et al., 2007; Park et al., 2010; Mclntyre et al., 2013 \& Osaili et al., 2013) and contradict with others such as Ansari-Lari et al., (2010) who do not believe in the role of food safety training to improve food handlers' knowledge; however, a possible explanation to such contradiction was provided by the study of Mclntyre et al. (2013) which was carried out on 698 food handlers in BC-Canada, where researchers found that knowledge of trained food handlers tends to descend over time.

\section{phase 2: Examination of the relationship between food safety knowledge of} $F \& B$ employees and their food handling practices

It was argued by Rennie (1995) that most courses of food safety training assume that in order to change food handling practices of any individual, s/he needs to be provided with knowledge. Nevertheless, this assumption was placed a part by many scholars (e.g., Howes et al., 1996; Ehiri et al., 1997 \& Griffith, 2000) who argued that training may succeed to increase individuals' knowledge, but this increase of knowledge does not necessarily create a change in their practices. To elucidate this debate, the current study examined the relationship between levels of food safety knowledge for F\&B employees and their food handling practices. Results emerged confirmed that the increase in food safety knowledge of F\&B employees is positively 
connected with a better food handling practices. This finding contradicts the aforementioned scholars' findings; but it consists with those of recently published studies (Acikel et al., 2008; Malhotra et al., 2008; Buccheri et al., 2010; Mclntryre et al., 2013 \& Sharif et al., 2013). It is apparent that the food safety training's role in improving food handling practices is still a debatable issue; this debate continuity suggests that other variables may influence the effectiveness of training in improving food handling practices. This research tried to find these variables by going through a third phase of investigation as discussed below.

\section{Phase 3: Exploring variables influencing the effectiveness of food safety training}

According to Tannenbaum and Yuki (1992), the majority of early research investigating training effectiveness were focusing on factors of training environment (e.g., training methods \& design). However, many other factors that are beyond the context of training may affect training effectiveness (Tracey \& Cardenas, 1996); these include supervisors' authorisation, availability of aids or constraints, supervisor and peer support (Seyler et al., 1998), availability of necessary facilities and resources (Clayton et al., 2002), motivation of management (Engel, 2001) and reinforcement programmes of trained skills (Walker et al., 2003). The current study employed a number of these factors: management motivation, availability of necessary facilities and resources and training reinforcement programmes. These factors revealed an intervening influence on the transformation of knowledge into practices by upgrading the correlation between food safety knowledge of $F \& B$ employees and their food handling practices as follows:

- Reinforcement programmes for trained skills upgraded correlation from $\mathrm{r}=0.484 * * *$ to $\mathrm{r}=0582 * * *$

- Management motivation upgraded correlation from $0.472 * * *$ to $0.582 * * *$

- Availability of necessary facilities and resources upgraded correlation from $\mathrm{r}=0.494 * * *$ to $\mathrm{r}=0.582 * * *$.

These findings confirmed the role of the new factors in enhancing the transformation of knowledge of food safety into a food handling practices (cf. Seaman \& Eves, 2006; Nieto-Montenegro et al., 2008 \& York et al., 2009). Furthermore, the results suggest that management motivation toward the implementation of learned knowledge and/or skills within the workplace plays the most powerful role in transferring trained skills into an improved food handling practices. 


\section{Limitations and future research}

A major limitation appeared during the review of literature was the scarcity of literature on food safety topics in Jordan and in other Arabic countries. Although a few researches discussing some food safety issues were found, most of them were microbiological ones. Another paucity which was existed is the lack of accurate foodborne outbreaks statistics in Jordan; this shortage of statistics restricted the ability of authors to describe the present food safety situation in Jordan. Nevertheless, the current research demonstrated pregnant findings including the confirmation of positive influence for food safety training on practices of food handlers; also, it adapted and examined the KAP model within the Jordanian context which is away from the western one where it was developed. The adapted KAP model represents a fruitful area for future researchers; they can apply it to investigate training's role in improving various types of behaviours within different contexts particularly those of food safety issues. The adapted KAP model provided new constructs (reinforcement programmes, management motivation \& availability of necessary resources \& facilities) that showed an intervening influence on food safety training effectiveness. However, there are still other factors including supervisor authorization, supervisor and peer support and availability of aids or constraints (Seyler et al., 1998) which have not been explored yet; accordingly, it is recommended to carry out future researches that examine similar factors to improve the explanatory power and validity of the KAP model.

\section{Conclusion}

The present study aimed to explore food safety training role in achieving a better food handling practices among F\&B employees working in hotels at the north of Jordan; this exploration was accomplished through the application of the KAP model's constructs within the aforementioned context. In Jordan, foodborne outbreaks occur each year; however, there is no estimation for these outbreaks (Osaili et al., 2013). In comparision to developed countries, Arab countries (among which is Jordan) were late in concerning of training as a tool of develpment; this is because their governments focused more in employing education to fill on the shortage of qualified workforce after the indepandance (Atiyyah, 1991). Based on the discussion of the previous three phases, it can be concluded that training of food safety is a useful technique for improving food handling practices with off-the-job (formal) training being more effective than on-the-job (informal) training. The amelioration of food handlers' practices was found to take place as a result of the evolve in their knowledge of food safety which positively correlates with these practices; such conclusion consists with existing studies (cf. Tebbutt, 1992; Kitcher, 1994; Kirby \& Gardiner, 1997 \& Sagoo et al., 
2003). Furthermore, the current study has delineated additional variables that can expand food safety knowledge transferred into food handling practices at workplace, including management motivation, availability of necessary facilities and resources needed to accomplish work-related tasks and programmes of reinforcing trained skills.

Findings of the current study contribute to the research body about food handlers' practices; particularly, the key contribution to the knowledge of food handling practices was the refinement of the KAP model by adding new constructs, defining conditions that facilitate the transmutation of knowledge into work-related behaviours; this refinement enhances the KAP model's ability in explaining food handlers' behaviour. These contributions to the knowledge were differentiated by shedding the light on food handlers' behaviour in the Jordanian and Arabic context; where there had been limited research effort spent on food handlers' behaviour. The present study contributed to the generalisation and usability of the KAP model (which has been established in the western context) in the global manner by validating it in the Arabic context.

Besides its knowledge contributions, the findings of this study have implications for different parties within the hotel industry and beyond it. First, Since training of food safety confirmed to have a positive influence on food handlers' practices, the Jordanian Food and Drug Adminstration (JFDA) push all food production organisations legaly (by food law of 2015) to train their employees on food safety issues (JFDA, 2017); however the JFDA imposes these organisations to train one food handler formaly who will be the informal trainer of her/his colleagues. Nevertheless, the superiority of formal training over the informal one has been illustrated by the current study's results; thus, the JFDA is recommended to consider formal food safety training more than the informal one. Second, foodservice managers face many challenges while controlling food safety such as their dependence on unskilled workforce with a high percentage of turnover (Shewmake \& Dillon, 1998); these challenges and others within the hospitality industry can be confronted by training (Tracey \& Cardenas, 1996). Food safety training is considered as a successful strategy to improve food handlers' knowledge and their work-related practices (Griffith, 2002 \& MacAuslan, 2003); this was confirmed in the present study's findings which also indicated to the superiority of formal training in improving food handling practices over the informal training. Accordingly, F\&B managers are recommended to arrange formal training programmes on food safety for their employees. Third, this study determined certain elements of workplace environment which enhance the transformation of knowledge and learned skills into proper food handling practices; these include management motivation, reinforcement programmes and availability of necessary facilities and resources. This finding entails that managers of $\mathrm{F} \& \mathrm{~B}$ establishments 
should enhance the aforementioned elements within their work environment in order to acquire better outcomes from employees' training. However, it was noted that managers of F\&B tend not to set up a supportive work environment; this can be attributed to the lack of managers' awareness for the importance and benefits of food safety training (Seaman \& Eves (2010). Accordingly, it is recommended to motivate F\&B managers for further support of food safety training implementation by educating them about its various benefits.

\section{References:}

1. Atiyyah, H. S. (1991). Effectiveness of management training in Arab countries. Journal of Management Development 10 (7), pp. 22-29.

2. Abdel-Dayem, M., Al Zou'bi, R., Bani Hani, R., and Amr, Z. S. (2014). Microbiological and parasitological investigation among food handlers in hotels in the Dead Sea area, Jordan. Journal of microbiology, Immunology and Infection, 47(5), pp. 377-380.

3. Abu-Madi, M. A., Behnke, J. M and Ismail, A. (2008). Patterns of infection with intestinal parasites in Qatar among food handlers and housemaids from different geographical regions of origin. Acta Tropica, 106(3), pp. 213-220.

4. Abu Al Saud, A. S. (1983). Faecal parasites in non-Saudi catering and domestic staff at the Ryadh military hospital. Saudi Medical Journal, 4(3), pp. 259-262.

5. Acikel, C., Ogur, R., Yaren, H., Gocgeldi, E., Ucar, M and Kir, T. (2008). The hygiene training of food handlers at a teaching hospital. Food Control, 19(2), pp. 186-190.

6. Ackerly, L. (1989). Food hygiene training. Food Magazine, 1(7), pp 22

7. Al-lahham, A. B., Abu-saud, M and Shehabi, A. A. (1990). Prevalence of Salmonella, Shigella and intestinal parasites in food handlers in Irbid, Jordan. Journal of Diarrhoeal Disease Research, 8(4), pp. 160162.

8. Altekruse, S. F., Yang, S., Timbo, B. B and Angulo, F. J. (1999). A multi-state survey of consumer food handling and food-consumption practices. American Journal of preventive Medicine, 16(3), pp. 216221.

9. Angelillo, I.F., Viggiani, N. M. A., Rizzio, L., and Bianco, A. (2000). Food handlers and food borne diseases: knowledge, attitudes and reported behaviour in Italy. Journal of food protection, 63(30), pp.381385.

10. Ansari-Lari, M., Soodbakhsh, S and Lakzadeh, L. (2010). Knowledge, attitudes and practices of workers on food hygienic practices in meat processing plants in Fars, Iran. Food Control, 21(1), pp. 260-263. 
11. Arif, A. E and Hassoun, A. S. (1969). An intestinal parasite survey among food handlers in Baghdad-1966. Bulletin Endemic Diseases, 11(1), pp. 7-27.

12. Aziri, B and Nedelea, A. (2013). Business strategies in tourism. ECOFORUM, 2(1-2), pp. 5-11. Available at: http://www.ecoforumjournal.ro/index.php/eco/article/view/21/12

13. Baldwin, T.T and Ford, J.K. (1988). Transfer of training: a review and directions for future research. Personnel Psychology, 41(1), pp. 63105.

14. Bryan, F. L. (1988). Risk of practices, procedures and processes that lead to outbreaks of food borne diseases. Journal of Food Protection, 51(8), pp. 663-673.

15. Buccheri, C., Mammina, C., Giammanco, S., Giammanco, M., La Guardia, M and Casuccio, A. (2010). Knowledge, attitudes and selfreported practices of food service staff in nursing homes and long-term care facilities. Food Control, 21(1), pp. 1367-1373.

16. Cavana, R., Delahye, B. and Sekaran, U. (2000). Applied business research: Qualitative and quantitative methods, NY: John Wiley and sons Australia, Ltd.

17. Clayton, D., Griffith, C., Price, P. and Peters, A. (2002). Food handlers' beliefs and self-reported practices. International Journal of Environmental Health Research, 12, pp. 25-39.

18. Cohen, J. (1988). Statistical power analysis for the behavioral sciences. $2^{\text {nd }}$ ed. Mahwah, NJ: Lawrence Erlbaum Associates.

19. Ehiri, J., Morris, G. and McEwen, J. (1997). Evaluation of a food hygiene training course in Scotland. Food Control, 8 (3), pp. 137-147.

20. Engel, D. (1998). Teaching HACCP-theory and practice from the trainer's point of view. Food Control, 9(2-3), pp. 137-139.

21. Engel, D. (2001). HACCP in training: food safety principles made easy'. London-UK. Chadwick House Group Ltd.

22. Food and Agriculture Organization (FAO)/World Health Organization (WHO). (2005). Knowledge, attitude and practice of food handling among housewives in Amman area", in Report of a Regional meeting on food safety for the Near East: Amman, Jordan. Available at: $\mathrm{ftp}$ ://ftp.fao.org/es/esn/food/meetings/NE_report_en.pdf

23. Foster, G. M. (1983). Socio-cultural practices affecting the safety of food. Food and Nutrition, 2(9), pp. 48-51.

24. Green, L. R and Selman, C. (2005). Factors impacting food workers' and managers' safe food preparation practices: qualitative study. Food Protection Trends, 25(12), pp. 981-990. 
25. Griffith, C. (2000). Food safety in catering establishments', in Farber, J. and Todd, E, (ed). Safe handling of foods. Marcel Dekker, Inc. NYUSA, pp. 235-256.

26. Griffith, C. (2002). Good practices for food handlers and consumers. In Blackburn, C. W and McClure, P. J, (ed). Food borne pathogens: Hazards, risk analysis and control, Woodhead Publishing Limited, Cambridge-UK, pp. 257-274.

27. Guthrie, R. K. (1988). Food sanitation. $3^{\text {rd }}$ ed. NY, USA.

28. Habiballah, M., Al-Shakhsheer, F and Al-Ababneh, M. (2017). Restaurant employees' food handling practices in Irbid city, Jordan. Journal of Tourism and Hospitality Management, 5(1), pp. 81-89.

29. Hendricks, K. J. and Layne, L. A. (1999). Adolescent occupational injuries in fast food restaurants: An examination of the problem from a national perspective. Journal of Occupational and Environmental Medicine, 41(12), pp. 1146-1153.

30. Hendry, C., Jones, A., Arthur, M. (1992). Skill Supply, training and development in the small-medium enterprise. International Small Business Journal, 10(1), pp. 68-72.

31. Hertzman, J and Barrash, D. (2007). An assessment of food safety knowledge and practices of catering employees. British Food Journal, 109(7), pp. 562-576.

32. Howes, M., McEwen, S., Griffith, M. and Harris, L. (1996). Food handler certification by home study: measuring changes in knowledge and behaviour. Dairy, Food and Environmental Sanitation, 16(11), pp. 737-744.

33. Imam, N. F. A., Abdulbaqi, Z. B and Fahad, R. A. (2016). The prevalence of intestinal parasitic infections among foreign workers in Madinah, Kingdom of Saudi Arabia. Saudi Journal of Medicine and Medical Sconces, 3(2), pp. 112-117.

34. Johns, N. (1995). Management food hygiene. $2^{\text {nd }}$ ed. London, UK. Macmillan Press.

35. Jones, P. and Lockwood, A. (2002). The management of hotel operations, Continium, London-UK.

36. Jones, P. (2004). Finding the hospitality industry? Or finding hospitality schools of thoughts?. Journal of Hospitality, Leisure, Sport and Tourism Education, 3(1), pp 33-45.

37. Jordan Investment Commission (JIC). (2015). Tourism sector, Available

at:

https://jic.gov.jo/portal/Services/JordanInvestmentCommission/Hotel s\%20and\%20Resorts

38. Jordanian Food and Drug Administration (JFDA). (2017). Food law of 2015. [Online]. 
http://www.jfda.jo/EchoBusV3.0/SystemAssets/PDF/AR/LawsAndR egulation/Nutrition-Food/FoodMain/29_304.pdf.(In Arabic).

39. Jordanian Ministry of Tourism and Antiquities (JMTA). (2017). Statistical newsletter 2017, [Online]. Available at: http://www.mota.gov.jo/Contents/Statistical_Summary_2017_.aspx

40. Khan, Z. A., Al-Jama, A. A and Madan, I. (1987). Parasitic infections among food handlers in Dammam and Al-Kobar", Saudi Arabia. Annals of Saudi Medicine, 7(1), pp. 47-50.

41. Khuri-Bulos, N. A., Abu Khalaf, M., Shehabi, A and Shami, K. (1994). Foodhandler-associated Salmonella outbreak in a university hospital despite surveillance cultures of kitchen employees. Infection Control and Hospital Epidemiology. 15, pp. 311-314.

42. Kirby, M.P. and Gardiner, K. (1997). The effectiveness of food hygiene training for food handlers. International Journal of Environmental Health Research, 7, pp. 251-258.

43. Kitcher, C. (1994). Case for food hygiene training'. Environmental Health, 102(6), pp 139-140.

44. Knowles, T. (2002). Food safety in the hospitality industry. Oxford, UK. Butterworth-Heinemann.

45. Luby, S. P., Jones, J and Horan, J. (1993). A large salmonellosis outbreak associated with a frequently penalized restaurant. Epidemiology and Infection, 110, pp. 31-39.

46. MacAuslan, E. (2003). Effective food hygiene training: a guide of owners and managers of food businesses, enforcement officers and potential trainers. Doncaster: Highfield.

47. Malhotra, R., Lal, P., Prakash, S., Daga, M and Kishore, J. (2008). Evaluation of a health education intervention on knowledge and attitudes of food handlers working in a medical college in Delhi, India. Asia-Pacific Journal of Public Health, 20(4), pp. 277-286.

48. Manning, C. K. (1994). Food safety knowledge and attitudes of workers from institutional and temporary foodservice operations. Journal of the American Dietetic Association, 94(8), pp 895-897.

49. Manpower Services Commission. (1981). Glossary of training terms. $3^{\text {rd }}$ ed. London: HMSO.

50. Mclntyre, L., Vallaster, L., Wilcott, L., Henderson, S and Kosatsky, T. (2013). Evaluation of food safety knowledge, attitudes and selfreported hand washing practices in FOODSAFE trained and untrained food handlers in British Colombia, Canada. Food Control, 30(1), pp. 150-156

51. Mortlock, M. P., Peters, A. C and Griffith, C. J. (1999). Food hygiene and Hazard Analysis Critical Control Point in the United Kingdom 
food industry: practices, perceptions, and attitudes. Journal of Food Protection, 62(7), pp. 786-792.

52. Mortlock, M.P., Peters, A.C., and Griffith, C. (2000). A national survey of food hygiene training and qualification levels in the UK food industry. International Journal of Environmental Health Research, 10, pp. 111-123.

53. Motarjemi, Y. and Käferstein, F. (1999). Food safety, Hazard Analysis and Critical Control Point and the increase in food borne diseases: a paradox?. Food Control, 10(4-5), pp 325-333. Elsevier Science Ltd.

54. Naing, N., Zain, M., Hamzah, W., Mat, H., Abdullah, N., Abu Bakar, M. (2007). A study on effectiveness of health education program on Knowledge, Attitude and Practice (KAP) of food handlers towards foodborne diseases and food safety. International Medical Journal, 14(4), pp. 253-260.

55. Nieto-Montenegro, S., Brown, J and LaBorde, L. (2008). Development and assessment of pilot food safety educational materials and training strategies for Hispanic workers in the mushroom industry using the health action model. Food Control, 19(6), pp. 616-633.

56. Osaili, T. M., Obeidat, B. A., Abu Jamous, D. O and Bawadi, H. A. (2011). Food safety knowledge and practices among college female students in north of Jordan. Food Control, 22(2), pp. 269-276.

57. Osaili, T. M., Abu Jamous, D. O., Obeidat, B. A., Bawadi, H. A., Tayyem, R. F and Subih, H. S. (2013). Food safety knowledge among food workers in restaurants in Jordan. Food Control, 31(1), pp. 145150.

58. Parish, M. E. (1998). Coliforms, Escherichia coli and Salmonella serovars associated with a cross-processing facility implicated in a Salmonellosis outbreak. Journal of Food Protection, 61(3), pp. 280284.

59. Park, S., Kwak, T. and Chang, H. (2010). Evaluation of the food safety training for food handlers in restaurant operations. Nutrition Research and Practice, 4(1), pp. 58-68.

60. Powell, S.C., Atwell, R.W., Massey, S.J. (1997). The impact of training on knowledge and standards of food hygiene-a pilot study. International Journal of Environmental Health Research, 7(4), pp. 329-334.

61. Pratten, J. and Curtis, S. (2002). Attitudes toward training in UK licensed retail: an exploratory case study. International Journal of Hospitality Management, 21, pp 393-403.

62. Ray, P. S., Bishop, P. A. and Wang, M. Q. (1997). Efficiency of the components of a behavioural program. International Journal of Industrial Ergonomics, 19, pp 19-29. Elsevier Science. 
63. Rennie, D. (1994). Evaluation of food hygiene education'. British Food Journal, 96 (11), pp. 20-25.

64. Rennie, D.M. (1995). Health education models and food hygiene education. Journal of Royal Society of Health, 115, pp. 75-79.

65. Sagoo, S. K., Little, C. L., Griffith, C. J., and Mitchell, R. T. (2003). Study of cleaning standards and practices in food premises in the UK. Communicable Disease and public Health, 6(1), pp. 3-4.

66. Seaman, P. and Eves, A. (2006). The management of food safety-the role of food hygiene training in the UK service sector. Hospitality Management, 25, pp 278-296.

67. Seaman, P and Eves, A. (2010). Perception of food hygiene training among food handlers, managers and training providers-a qualitative study. Food Control, 21(1), pp. 1037-1041.

68. Seyler, D. L., Holton III, E. F., Bates, R. A., Burnett, M. F. and Carvalho, M. A. (1998). Factors affecting motivation to transfer training. International Journal of Training and Development, 2(1), pp. 2-16.

69. Shapiro, R., Ackers, M., Lance, S., Rabbani, M., Schaefer, L., Daugherty, J., Thelen, C and Swerdlow, D. (1999). Salmonella Thompson associated with improper handling of roast beef at a restaurant in Sioux Falls, South Dakota. Journal of Food Protection, 62(2), pp. 118-122.

70. Sharif, L., Obaidat, M. M and Al-Dalalah, M. (2013). Food hygiene knowledge, attitudes and practices of the food handlers in the military hospitals. Food and Nutrition Sciences, 4(3), pp. 245-251.

71. Shewmake, R. A and Dillon, B. (1998). Food poisoning: causes, remedies, and prevention. Postgraduate Medicine, 103(6), pp. 125129, 134, 136.

72. Simsek, Z., Koruk, I., Copur, A. C and Gurses, G. (2009). Prevalence of staphylococcus aureus and intestinal parasites among food handlers in Sanliurfa, Southeastern Anatolia. Journal of Publich Health Management and Practice, 15(6), pp. 518-523.

73. Sinclair, R., Smith, R., Colligan, M., Prince, M., Nguyen, T. and Stayner, L. (2003). Evaluation of safety training program in three food service companies. Journal of Safety Research, 34, pp. 547-558.

74. Smith, R. (1994). Food hygiene training: the chance to create a coherent policy. British Food Journal, 96(7), pp 41-45.

75. Tannenbaum, S. I and Yuki, G. (1992). Training and development in work organizations. Annual Review of Psychology, 43, pp. 399-441.

76. Tebbutt, G. M. (1992). An assessment of food-hygiene training and knowledge among staff in premises producing or selling high-risk 
foods. International Journal of Environmental Health Research, 2(3), pp 131-137.

77. Tones, K. and Tilford, S. (1994). Health education: effectiveness, efficiency and equity. $2^{\text {nd }}$ ed. Chapman and Hall. London-UK.

78. Tracey, J. and Cardenas, C. (1996). Training effectiveness: an empirical examination of factors outside the training context. Hospitality Research Journal, 20(2), pp 113-123.

79. Tracey, J. B., Tannenbaum, S. I. and Kavanagh, M. J. (1995). Applying trained skills on the job: the importance of the work environment. Journal of Applied Psychology, 80(2), pp 239-252.

80. Vought, K. J and Tatini, S. R. (1998). Salmonella enteritidis contamination of ice cream associated with a 1994 multistate outbreak. Journal of Food Protection, 61(1), pp. 5-10.

81. Wakid, M. H. (2006). Distribution of intestinal parasites among food handlers in Jeddah, Saudi Arabia, Journal of Parasitic Diseases, 30(2), pp. 146-152.

82. Walker, E., Pritchard, C and Forsythe, S. (2003). Food handlers' hygiene knowledge in small food businesses. Food Control, 14(5), pp. 339-343.

83. Walter, A., Cohen, N. and Swicker, R. (1997). Food safety training needs exist for staff and consumers in a variety of community-based homes for people with the developmental disabilities. Journal of the American Dietetic Association, 97(6), pp. 619-625.

84. Wilson, M. D. J., Murray, A. E., Black, M. A and McDowell, D. A. (1998). Training and education in contract catering management. Journal of European Industrial Training, 22(2), pp. 73-81.

85. Worsfold, D. (2005). A survey of food safety training in small food manufacturers. International Journal of Environmental Health Research, 15(4), pp. 281-288.

86. Worsfold, D. and Griffith, C. (2003). A survey of food hygiene and safety training in the retail and catering industry. Nutrition and Food Science, 33(2), pp. 68-79.

87. Worsfold, D., Griffith, C. and Worsfold, P. (2004). A survey of environmental health officers' views of food hygiene training. British Food Journal, 106(1), pp. 51-64.

88. York, V., Brannon, L., Shanklin, C., Roberts, K., Barrett, B., and Howells, A. (2009). Interventions improve restaurant employees' food safety compliance rates. International Journal of Contemporary Hospitality Management, 21(4), pp.459-478. 